\title{
The Method of Multi-attributeTarget Recognition Based On Laser Detection
}

\author{
CHEN Jian-biao ${ }^{1, a}$,SUN Hua-yan ${ }^{2}$ and ZHENG Yong-hui ${ }^{1}$ \\ ${ }^{1}$ Company of Postgraduate Management, Academy of Equipment, Beijing 101416,China \\ ${ }^{2}$ Department of Photoelectric Equipment, Academy of Equipment, Beijing 101416,China \\ ageneral_chen2041@163.com
}

Keywords: Laser Detection; Target Recognition; High Range Resolution Profile; Multi-attribute Extraction.

\begin{abstract}
Ideal result of target recognition can't be obtained just with single attribute. In order to improve the performance of target recognition and reflect the physical attributes of the target, some attributes are extracted from the high range resolution profile of the unknown target to form an multi-attribute vector, such as the frequency amplitude, the length of energy gathering, the centre moments and the bispectrum and so on.Because of the high dimensions of the vector, the PCA (Principal Component Analysis) method is used to decrease the dimension. The processed vector is regarded as the input of the BP (Back Propagation) neural network to obtain the result of target recognition.The simulation result indicates that it is helpful for improving the target recognition rate with the multi-attribute extraction method and PCA method.
\end{abstract}

\section{Introduction}

The high range resolution profile[1] (HRRP) of the target is the projection of the two-dimension profile towards to the detection orientation. It can be used to identify unknown target because the HRRP contains much structure information about the target.Originally the HRRP was used in the domain of radar target recognition[2]. It also can be used in laser detection technology[3].

The resolution of the system is very high when the pulse width of the laser is narrow. The complex target can be divided into some range cells, so the strong scattering points of the target can be extracted. The fluctuant echo of the target forms the HRRP[4]. But the HRRP is sensitive to the variation of attitude angle, the distance between the target and the detection system and target translation. Therefore the attributes that are not sensitive to these variations are selected[5]. The frequency spectrum and the centre moments are transaction invariant features. And the bispectrum[6, 7] of the HRRP is not sensitive to the angle. At the same time the length of energy gathering can reflect the length of the target approximately.

The principal component analysis method is used to decrease the dimension of the attribute vector, it is based on the multi-element statistic analysis technique. This method can exclude the overlap section of the features, meanwhile it keeps the completeness of the original information.

In this paper, the BP neutral network[8]is also used to recognize target. It has good learnability and self-organization, and it can be trained by different methods. So it is appropriate to be used in target recognition domain.

\section{Multi-attribute of HRRP}

Frequency Spectrum.In order to eliminate the translation sensitivity, the translation invariant feature is selected. The frequency spectrum loses the total phase features, but it holds good as a good feature for target recognition. It is the amplitude of the Fourier Transform of the target HRRP and indicates the distribution of the energy of every frequency. Assuming that $X(n)$ is real HRRP of target, then the frequency spectrum of it can be expressed as:

$$
A(w)=\operatorname{abs}(f f t(X(n))), n=0,1,2, \ldots, N-1
$$


In this formula,fft stands for the Fourier Transform; abs is on behalf of the operation absolute value; $n$ is the nth component of the range profile.

Length Of Energy Gathering.The length of energy gathering can reflect the radial length of the target approximately, so it can be as a feature to recognize the target. Assuming that in the domain of a certain angle, $x(i)$ is a discrete serie of the ith HRRP, and $x(i, j)$ indicates the amplitude of thejthsampling points, $j=1,2, \ldots, N$ therein. The specific method of extracting the length of energy gathering is as follow:

1)calculating the mean of $x(i)$

$$
\bar{x}(i)=\frac{1}{N} \sum_{j=1}^{N} x(i, j)
$$

2)setting an threshold value $T$

$$
T=\eta * \bar{x}(i)
$$

In this formula, $\eta$ is the confidence coefficient, its value is between 0.8 and 1 , it depends on the SNR of the HRRP. When the SNR is high, its value can be little, vice versa.

3)The value of $x(i)$ is searched from both ends, the searching stops when the first point appears respectively at both ends and note down their location as $m(i), n(i)$, then the length of energy gathering is :

$$
L(i)=n(i)-m(i)
$$

Centre moment. $\{x(n), n=1,2, \ldots, N\}$ is the HRRP,and $\mathrm{n}$ is the number of the range elements. Through normalizing as follow :

$$
\bar{x}(n)=\frac{x(n)}{\sum_{n=1}^{N} x(n)}
$$

$\bar{x}(n) \in[0,1]$, and

$$
\sum_{n=1}^{N} \bar{x}(n)=1
$$

so $\bar{x}(n), n=1,2, \ldots, N$ can be regarded as a discrete distribution function. The $p$ thcentre moment of range profile $\bar{x}(n)$ is defined as :

$$
\mu^{(p)}=\sum_{n=1}^{N}\left(n-n_{0}\right)^{p} \bar{x}(n)
$$

Therein,

$$
n_{0}=\sum_{n=1}^{N} n \bar{x}(n)
$$

is the first origin moment, namely the scattering center of the HRRP.

Because of $\mu^{(1)} \equiv 0$, the $2 \sim$ pth centre moments are selected to form the feature vector of $\left(p_{\max }-1\right)$ dimensions: 


$$
f=\left[f(1), f(2), \ldots f\left(p_{\max }-1\right)\right]^{T}=\left[\mu^{(2)}, \mu^{(3)}, \ldots, \mu^{p_{\max }}\right]^{T}
$$

$p_{\max }$ is the maximum value of the centre moments, which value is selected of $p_{\max }$ depends on experience. Because the amplitude of thecentre moments increases quickly along with centre moment rank, the weights of different moments are different. So $X \max -X \min$ transformation is acted on $\mathrm{f}$ for eliminating the effect of dimension. After the transformation , every dimension has the same weight.

$$
\overline{f_{i}}=\frac{f_{i}-f_{i, \min }}{f_{i, \text { max }}-f_{i, \min }}, i=1,2, \ldots, p_{\max }
$$

Therein, $f_{i, \min }$ is the minimum value of all centre moments, $f_{i, \max }$ is the maximum value of all centre moments. Along with the increment of the dimension, thecentre moments will have redundant information. It is not helpful for recognizing target, so the PCA technique is used to decrease the dimension of the feature vector.

Bispectrum.The definition of bispectrum comes from the high order cumulant. Assuming that $\{x(k)\}$ is a real discrete stationary random sequence, its mean is zero. The three order cumulant is :

$$
\operatorname{Cum}(x(k), x(k+m), x(k+n))=E\{x(k) x(k+m) x(k+n)\}{ }^{\Delta} C_{3 x}(m \cdot n)
$$

If the three order cumulant is absolute summable, the discrete Fourier Translation of it is called the bisepctrum of the series. Namely,

$$
B\left(\omega_{1}, \omega_{2}\right)=\sum_{-\infty}^{\infty} \sum_{-\infty}^{\infty} C_{3 x}(m, n) e^{-j\left(\omega_{1} m+\omega_{2} n\right)}
$$

The bispectrum is of periodicity and symmetry. So the bispectrum of the total plane of $\omega_{1}-\omega_{2}$ can be obtained, if only the triangular domain is calculated: $\omega_{1} \geq 0, \omega_{1} \geq \omega_{2}, \omega_{1}+\omega_{2} \leq \pi$. Therefore, the bispectrum can be a feature for target recognition.

\section{Principal Component Analysis Method}

In this paper an important poin is how to extract the attributes that can reflect the features of the HRRP. The frequency spectrum is selected to describe the feature in frequency domain. And the length of energy gathering can identify the targets that are very different in real size. The centre moments are translation invariant and the bispectrum is not sensitive to the variation of the target's attitude angle. The feature vector is comprised of the attributes mentioned before. It is obvious that the dimension of the feature vector is very high. It is very complicated and difficult for the recognition algorithm. So the PCA[9] method is adopted to decrease the dimension of the feature vector.

The PCA is used to analyse the dataset that is zero-mean. Assuming that the dataset is a $M \times N$ matrix $X=\left\{x_{1}, x_{2}, \ldots, x_{n}\right\}, \quad x_{i}, \quad i=1,2,3, \ldots, \quad$ is a $M$ dimensional column vector. Then

$$
\frac{1}{N} \sum_{i=1}^{N} x_{i}=0
$$

The covariance matrix of dataset is defined as: 


$$
C=\frac{1}{N} \sum_{i=1}^{N} x_{i} X_{i}^{T}
$$

The PCA method is used to process eigen decomposition of the covariance matrix.

$$
\lambda v=C v
$$

Then the eigenvalues are sorted by size $\lambda_{1} \geq \lambda_{2} \geq \ldots \geq \lambda_{n}$, the first $Q$ eigenvalues and their eigenvector set are obtained $U=\left[u_{1}, u_{2}, \ldots, u_{q}\right]$, and $U$ is called sub-feature space. $Q$ represents the number of the principal components. Thevalue of $\mathrm{Q}$ can be determined by:

$$
\sum_{i=1}^{q} \lambda_{i} / \sum_{i=1}^{n} \lambda_{i} \geq b
$$

Generally, $b$ is between zero and one.

\section{Recognition Method of BPNN}

The BPNN[10, 11] (Back Propagation Neural Network ) is a neural network of back propagation which is consisted of the input layer, the hidden layer and the output layer. Every layer is connected with each other. The number of the hidden layer can be one or more. The learning method is teacher learning. In this process, every input pattern is corresponding to an expected output. The error is the difference between the input and the expected output. According to the principle of minimum error sum of squares, the connected weights and the threshold values are modified from the output layer to the hidden layer. This course is called back propagation of error. And this process stops when the error is acceptable. Finally, the output of the BPNN is very approximate to the expected values. The neuron is the basic element of neural network, the transfer function is tansig in the hidden layer and logsig in the output layer. The picture below in Fig.1 presents the typical neural network of three layers.

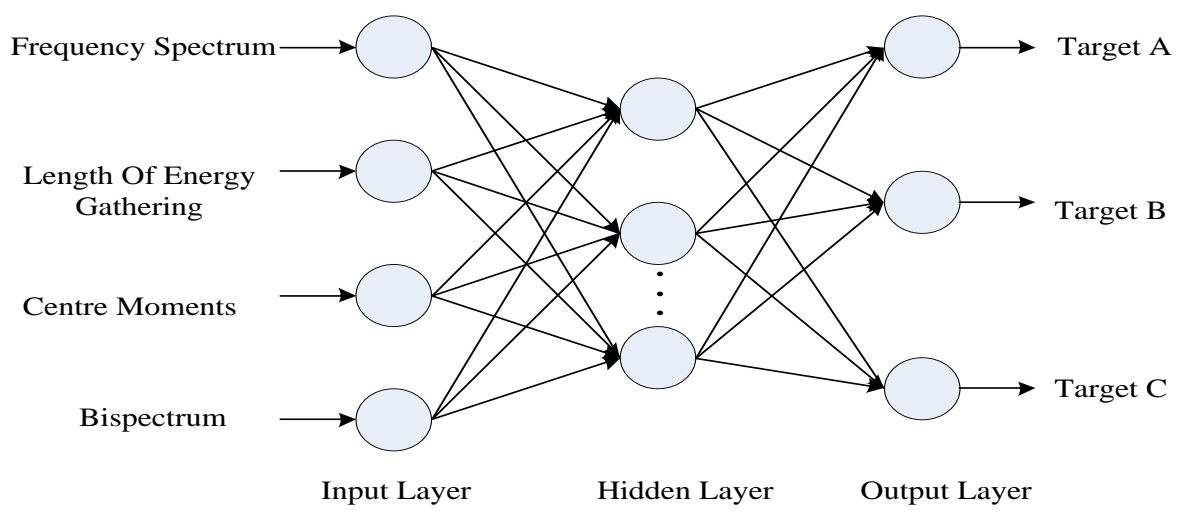

Fig.1.Structure of the three layers BPNN

The number of the layers and neurons in every layer depends on the real condition, and the project of training the neutral network is necessary and important. So selecting the appropriate training function is the key step.

\section{Simulation}

The HRRPs of three targets are simulated by MATLAB. The object resemblingcylinder represents the missile. And thecone and sphere are instead of the false targets. When the detection range is invariant, a number of HRRPs are obtained. The value of the pitch angle is $80,90,100$ 
degree and the range of the azimuth is from -130 to -140 degree. The four features mentioned before are processed by PCA method. Then the feature vector is regarded as the input of the BPNN. The output of the BPNN stands for the category of the targets.

In the simulation, the three-layer BPNN is selected. The input layer is of uncertain neurons which are corresponding to different features. And the output layer is of three neurons which are corresponding to three kinds of targets. The number of the neurons of the hidden layer depends on the number of neurons of the input layer and the output layer. Generally, the number of the neurons of the hidden layer is more and the precision of the result is higher. But it will cost a lot of time to train the network and make the network unstable. Therefore, after considering all the factors the number of the neurons of the hidden layer is selected as fifteen. The 75 percent of the data is used to train the BPNN, and the rest is used to test the network.The multi-attribute used in simulation is comprised of four features mentioned before.The HRRPs of three targets are showed in the Fig.2. And the frequency spectrum of the HRRPs is given in Fig.3.The contour maps of bispectrum extracted from HRRP are shown in Fig 4.Then the result of recognition is presented in Table 1.

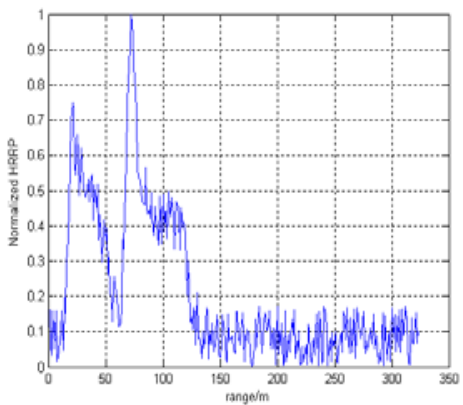

(a)Missile analogue

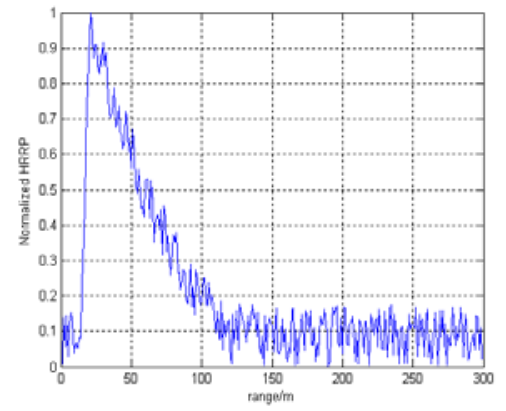

(b)Interference object I (c) Interference object II

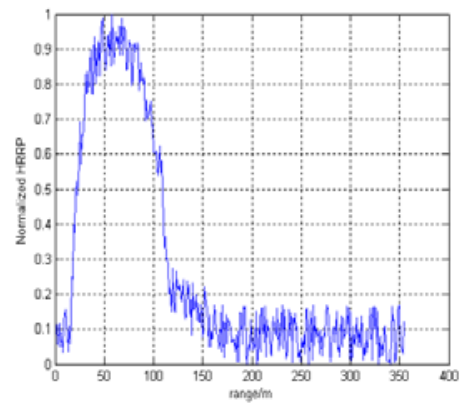

Fig.2. The HRRP of three targets when angle of pitch is $80^{\circ}$ and the azimuth is $-130^{\circ}$.

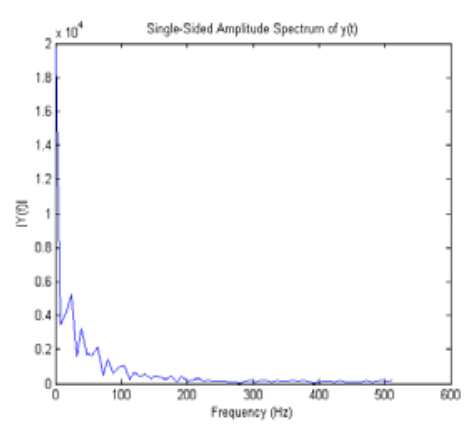

(a) Missile analogue

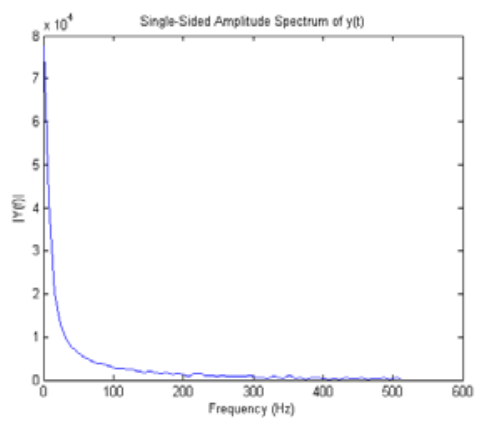

(b) Interference object I

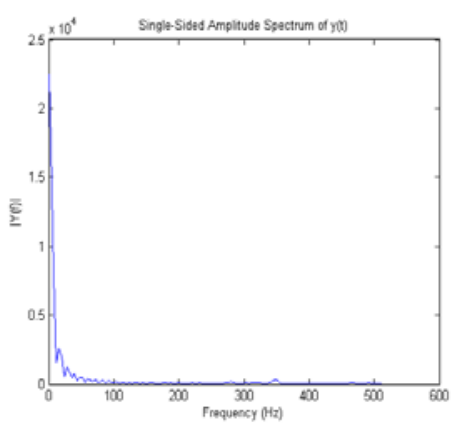

(c) Interference object II

Fig.3. The frequency spectrum of HRRP

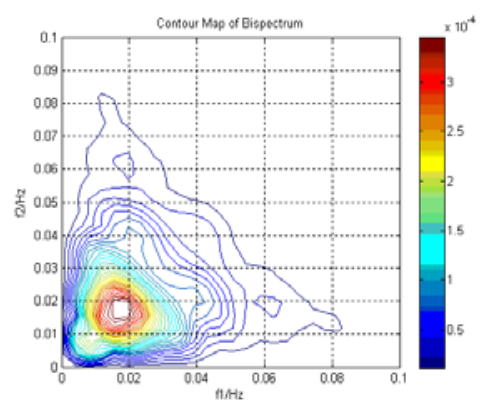

(a) Missile analogue

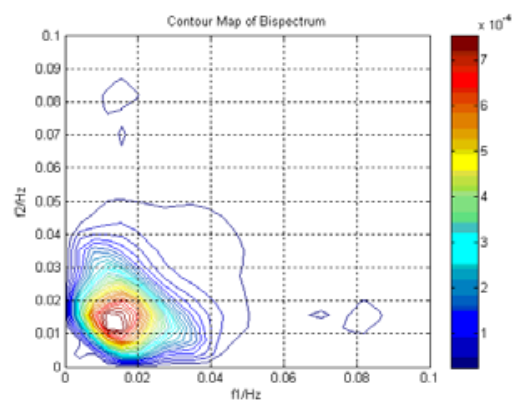

(b) Interference object I

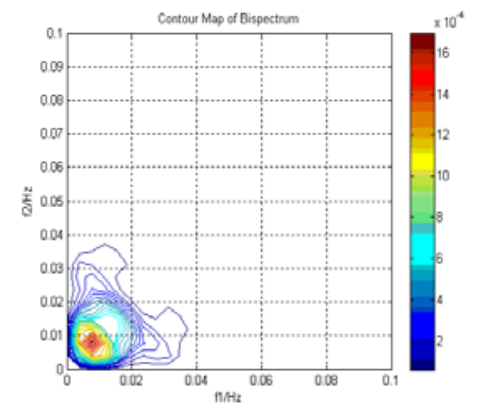

(c) Interference object II

Fig.4. The contour map of bispectrum extracted from HRRP 
Table 1 Result of Recognition

\begin{tabular}{ccc}
\hline Feature Extracted & Rate of Right Recognition/\% & Dimension of feature \\
\hline Frequency spectrum & 78.31 & 64 \\
Length of energy gathering & 51.63 & 1 \\
Centre moments & 56.78 & 5 \\
Bispectrum & 69.58 & 128 \\
Multi-attribute & 89.69 & 56 \\
\hline
\end{tabular}

\section{Conclusion}

This paper presents a method of multi-attribute target recognition based on the HRRP. Four features are extracted from the HRRP to comprise of the feature vector. The PCA method is used to compress the data of the feature vector. And the BPNN is used to recognize the unknown targets.According to the result of simulation, the right rate of recognition is the highest using the multi-attribute.

But the recognition effect of the features extracted from HRRP can be improved more and the efficiency of the BPNN is not enough. Therefore, in the next step improving the effect of the recognition algorithm is an important work.

\section{References}

[1] Schoemaker R, Benoist K. Characterisation of small targets in a maritime environment by means of laser range profiling[Z]. 2011.

[2] CUI Yanping, GE Xingwei, Recognition of Rotary Objects from Complex Background [J].Semiconductor Optoelectronics: 2010(06): 931-935.

[3] Kim K, Seo D, Kim H. Efficient radar target recognition using the MUSIC algorithm andinvariant features[J]. IEEE Transactions on Antennas and Propagation. 2002, 50(3): 325-337.

[4] Lei Yang, Xiaodan Wang and Yuxi Zhang. A Radar Target Recognition Approach Based onMulti-Feature Extraction[J]. Electronics Optics \& Control. 2011, 18(12): 35-38.

[5] Wong S K. Distorting effects on the high-range-resolution profile of a target due to small random rotational motion of the target[Z]. 1998: 3462.

[6] HongJ, Jie L, Weixin X, et al. Bispectrum-based radar target classification[Z]. 1998:419-422.

[7] Tague C, Sullican E J. Bispectrumdetection of nonstationary signals:new theoretical results[Z]. 1991.

[8] SUN Honghui, WANG Hongxia and TIAN Tao. The recognition method of objects based on moment invariant and BP neural network [J].Microelectronics and computer. 2011(03): 63-65.

[9] WEN Fuxi, LIU Hongwei. Space target recognition based on principal component analysis [J]. Applied science and technology. 2007(01): 1-4.

[10] CHEN Shengang, XU Teng. Aerial Target Identification Based On BP Neural Network [J]. Command control and simulation. 2007(03): 30-32.

[11] CAO Wei, ZHOU Hui. High resolution radar target recognition based on multi-dimensional feature vector and BP network [J]. Computer engineering and applications. 2013(08): 213-216. 